\title{
1 Assessing dose rate distributions in VMAT plans
}

2

3
P-H Mackeprang, W Volken, D Terribilini, D Frauchiger, K Zaugg, D M Aebersold, M K Fix and P Manser

Division of Medical Radiation Physics and Department of Radiation Oncology, Inselspital, Bern University Hospital, and University of Bern, Switzerland

E-Mail: paul-henry.mackeprang@insel.ch

Abstract. Dose rate is an essential factor in radiobiology. As modern radiotherapy delivery techniques such as Volumetric Modulated Arc Therapy (VMAT) introduce dynamic modulation of the dose rate, it is important to assess the changes in dose rate. Both the rate of monitor units per minute (MU rate) and collimation are varied over the course of a fraction, leading to different dose rates in every voxel of the calculation volume at any point in time during dose delivery.

Given the radiotherapy plan and machine specific limitations, a VMAT treatment plan can be split into arc sectors between DICOM Control Points of constant and known MU rate. By calculating dose distributions in each of these arc sectors independently and multiplying it with the MU rate, the dose rate in every single voxel at every time point during the fraction can be calculated. Independently calculated and then summed dose distributions per arc sector were compared to the whole arc dose calculation for validation. Dose measurements and video analysis were performed to validate the calculated datasets. A clinical head and neck, cranial and liver case were analyzed using the tool developed.

Measurement validation of synthetic test cases showed linac agreement to precalculated arc sector times within $\pm 0.4 \mathrm{~s}$ and doses $\pm 0.1 \mathrm{MU}$ (one standard deviation). Two methods for visualization of dose rate datasets were developed: The first method plots a 2D histogram of the number of voxels receiving a given dose rate over the course of the arc treatment delivery. In similarity to treatment planning system display of dose, the second method displays dose rate as color wash on top of the corresponding CT image, allowing the user to scroll through the variation over time. Examining clinical cases showed dose rates spread over a continuous spectrum, with mean dose rates hardly exceeding $100 \mathrm{cGy} / \mathrm{min}$ for conventional fractionation. A tool to analyze dose rate distributions in VMAT plans with sub-second accuracy was successfully developed and validated. Dose rates encountered in clinical VMAT test cases show a continuous spectrum with a mean less than or near $100 \mathrm{cGy} / \mathrm{min}$ for conventional fractionation. 


\section{Introduction}

The main quantity for radiotherapy efficacy is deposited energy per unit mass, measured in units of Gray. Cell kill in vitro and tumor response in patients have clearly been shown to be linked to the dose applied. Biological response to radiotherapy dose rates, on the other hand, is a subject of both controversy and ongoing research (Ling et al 2010). When taking into account the modulation modern radiotherapy techniques such as VMAT have brought to radiotherapy delivery, two different factors need to be distinguished: On one hand, the treatment machine modulates its output, changing the number of monitor units (MU) per minute (MU rate). On the other hand, every voxel in the calculation volume receives a different dose per time interval, which is referred to as dose rate. Compared to constant dose rates, even less is known about the effects of modulated dose rates. In patient applications, four different orders of magnitude may be examined in radiotherapy dose rate (Murphy et al 2007): fractionation schemes in the order of weeks, fraction delivery time lasting minutes, intrafraction modulation from second to second and lastly linear accelerator beam generation in microsecond pulses. Of these four, fractionation schedules have been examined empirically to the greatest extent. Data on intrafraction dose rate, however, hasn't been as comprehensive yet (Ling et al 2010).

In the past, in vitro studies have focused on the effect of protracted dose delivery (lowered dose rate) or treatment interruptions. According to experimental data (Mu et al 2003, Wang et al 2003, Fowler et al 2004, Shibamoto et al 2004, Ogino et al 2005, Joiner et al 2010) fractions lasting longer than 20-30 min have to be considered less effective in terms of cell kill than fractions shorter than that. In a first model based approach (Altman et al 2006), a simplified IMRT model showed possibilities for increased cell kill when rearranging the IMRT field order especially with increasing fraction delivery time. Bewes et al. (Bewes et al 2008) confirmed this evidence, but also suggested that "regional variation in the dose rate across a tumor [...] will affect dose efficacy". Although in vitro experiments showed differences in cell kill in the setting of fixed and known dose rates for Co60 irradiations (Hall 1972), the actual temporal and spatial distribution of dose rates during a modulated radiotherapy fraction in situ remains unknown.

Both IMRT and VMAT use modulation of the MU rate to achieve conformal dose delivery to a tumor and to spare surrounding organs at risk. At any time, only a small part of the target volume is irradiated with the maximum MU rate chosen in the plan. Intrafraction dose rate depends on the same factors affecting dose distribution in tissue, e.g. MU rate, field shape, leakage, tissue composition, distance to source and radiation quality (photons or electrons and their respective energies). It has been proposed by others to analyze these spatiotemporal patterns of dose rate modulation, albeit with a resolution of minutes of radiotherapy delivery (Altman et al 2006, Murphy et al 2007). As evidence of immediate radiation response initiating DNA damage repair within seconds (Ponette et al 2000) and high dose rate treatments (Favaudon et al 2014) grows though, the need for a comprehensive analysis of dose rates emerges.

This study shows a method to calculate and visualize planned VMAT dose rate distributions in 3D and time using MC techniques with sub-second resolution.

\section{Material and Methods}

\subsection{Dose Rate Calculation}

In this work, the Swiss Monte Carlo Plan (SMCP) framework (Fix et al 2007) was used for all MC dose calculations. Connected to the Eclipse treatment planning system (TPS), the SMCP allows easy integration of a powerful modular MC dose calculation framework offering the choice of several 
currently available particle transport codes. Input to the system may be via the TPS or a proprietary input file format, a combination of which was used in this study.

Calculating a dose rate distribution extends the SMCP by the time domain: a dose known to be applied with constant MU rate and the corresponding delivery time are needed. In VMAT treatments, two consecutive DICOM control points (CPs) form one arc sector. As regulated in the DICOM standard, in both IMRT and VMAT treatment, the MU rate is set at each DICOM control point for the following arc sector by the linac. In the system used, the resulting delivery parameters, such as arc sector weight, MU rate in MU per minute, gantry speed and MU per degree are precalculated by the TPS and used by the developed tool. The TPS calculates these parameters taking into account linac specific limitations, e.g. maximum gantry rotation speed, maximum MLC leaf speed or maximum MU rate as set in the plan. Within clinical tolerances, these constraints are assumed to be met by the machine.

Starting from an anonymized VMAT DICOM RT plan file, data is read into the TPS for precalculation of MU rates and arc sector times as detailed above. Meanwhile, the same plan file is converted to the proprietary SMCP file format and split into separate files between two consecutive $\mathrm{CPs}$, corresponding to one arc sector each. Any of these files contain all information needed for dose calculation. Typical cases include 100 to 200 CPs per VMAT arc, resulting in a dose delivery time of less than $0.5 \mathrm{~s}$ per arc sector. Dose calculations are then performed independently using these files as input, each representing a period of constant MU rate, but dynamic beam angle and field shape. In SMCP, results of the MC calculation are stored in units of cGy per MU (Which can directly be used to calculate the dose rate). By multiplying with the precalculated (constant) MU rate in units of MU per minute, the tool can then store the data as dose rates applied between two CPs for each voxel.

\subsection{Validation}

Both the dose rate calculations as well as their visualization were validated with test cases and against measurements.

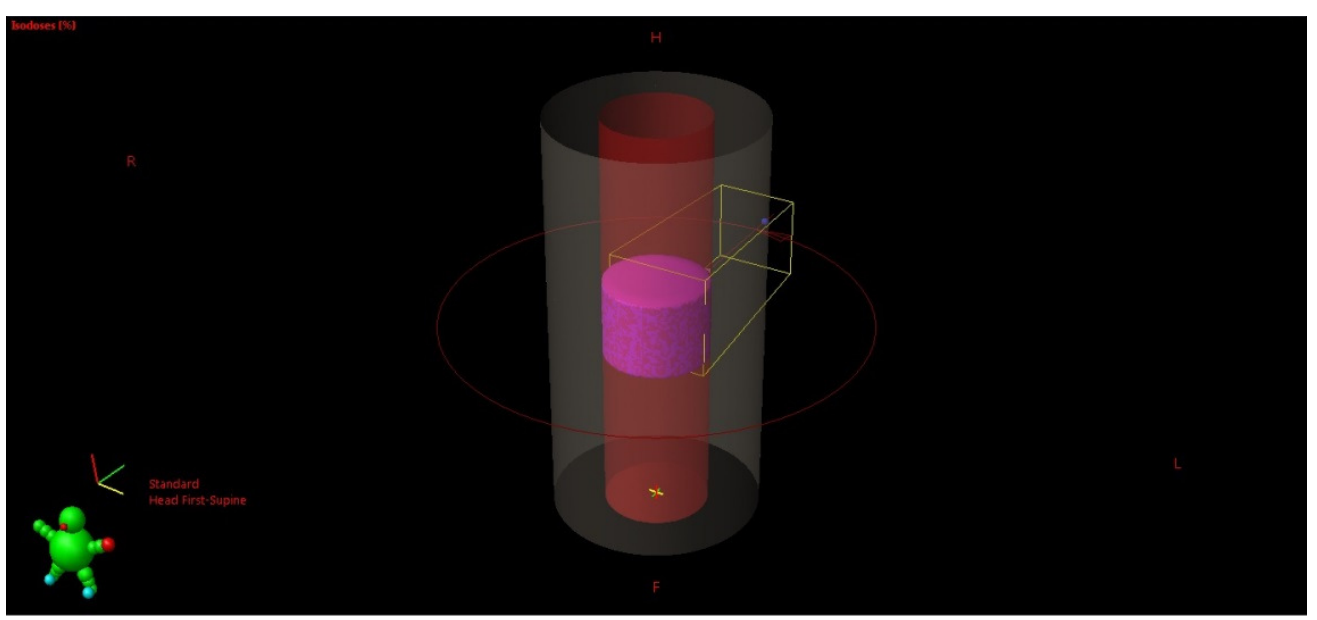

Figure 1: Schematic view of the cylindrical homogeneous water phantom with a diameter of $20 \mathrm{~cm}$ used for the validation of dose rate calculations.

Artificial test cases were defined in SMCP to validate the dose rate calculations. As a first test case, a virtual cylindrical water phantom with a diameter of $20 \mathrm{~cm}$ as shown in figure 1 was generated to create test plans with a fixed source to surface distance to mimic reference conditions as defined for static fields (Almond and Biggs 1999). This phantom was used for dose rate calculations for artificial plans, manually varying the MU rate over the course of an arc in the plan file. Table 1 shows the settings used for three test cases. 
1 Table 1. Characteristics of artificial treatment plans of a cylindrical homogeneous water phantom 2 with a diameter of $20 \mathrm{~cm}$ used to validate machine compliance to the precalculated MU rates.

\begin{tabular}{llllllll} 
Plan & arc sector & 1 & 2 & 3 & 4 & 5 & 6 \\
\hline 1 & Gantry angle & $0-72^{\circ}$ & $72-144^{\circ}$ & $144-216^{\circ}$ & $216-288^{\circ}$ & $288-360^{\circ}$ & \\
& MU & 600 & 500 & 400 & 300 & 200 & \\
& MU rate (MU/min) & 600 & 600 & 600 & 600 & 600 & \\
\hline 2 & Gantry angle & $0-72^{\circ}$ & $72-144^{\circ}$ & $144-216^{\circ}$ & $216-288^{\circ}$ & $288-360^{\circ}$ & \\
& & & & & & & \\
& MU & 300 & 100 & 300 & 1000 & 300 & \\
& MU rate (MU/min) & 600 & 400 & 600 & 600 & 600 & \\
\hline 3 & Gantry angle & $0-60^{\circ}$ & $60-120^{\circ}$ & $120-180^{\circ}$ & $180-240^{\circ}$ & $240-300^{\circ}$ & $300-360^{\circ}$ \\
& & & & & & \\
& MU & 125 & 125 & 375 & 125 & 125 & 125 \\
& MU rate (MU/min) & 600 & 600 & 600 & 600 & 600 & 600 \\
\hline
\end{tabular}

3

4 These test cases as well as the clinical head and neck case were used to validate the machine 5 compliance to the precalculated MU rates and behavior when switching from one arc sector of fixed

6 MU rate to the next. Plans were calculated in arc sectors, and resulting dose rate distributions were 7 checked manually. To preclude calculation errors, arc sector dose rate distributions were multiplied 8 with their respective application time, summed up and compared against the static Monte Carlo dose 9 calculation.

10 Measurements were conducted to validate accurate delivery of the synthetic test cases. Plans 11 were delivered on a Varian Novalis Tx linac to a Delta4 phantom and doses compared arc sector by 12 arc sector. MU rates were recorded from the linac control panel and compared to the values 13 precalculated by the TPS and used as input to the SMCP.

\section{2.3. Application}

15 Three clinical test cases were chosen: A conventionally fractionated head and neck case planned with 4 VMAT arcs, a cranial non-coplanar 4 arc case and a hypofractionated 2 arc liver case.

All calculations were performed using MC as transport code to a statistical uncertainty of about $1 \%$ using $0.25 \mathrm{~cm}$ edge length calculation grid voxels. Table 2 summarizes the treatment plan parameters for each of the clinical test cases, with application time describing the time from beam on to beam off of each arc. Maximum MU rate was set to $600 \mathrm{MU}$ per minute in all cases. All plans were calculated and dose rate distributions analyzed using the visualization methods described below. Mean dose rate was calculated as arithmetic mean of all voxels at all time points over one arc within a volume of interest. 
1 Table 2. Plan characteristics for three clinical VMAT treatment cases selected for dose rate analysis.

\begin{tabular}{|c|c|c|c|c|c|}
\hline Test case & $\begin{array}{l}\text { Prescribed } \\
\text { fraction dose } \\
(\mathrm{Gy})\end{array}$ & Technique & $\begin{array}{l}\text { MU } \\
\text { per arc }\end{array}$ & $\begin{array}{l}\text { Number of arc } \\
\text { sectors }\end{array}$ & $\begin{array}{l}\text { Application time } \\
\text { (s) }\end{array}$ \\
\hline \multirow[t]{4}{*}{ Head and neck } & 2 & 4 VMAT $\operatorname{arcs}$ & 162 & 113 & 41.5 \\
\hline & & & 184 & 97 & 36.2 \\
\hline & & & 309 & 177 & 74.6 \\
\hline & & & 197 & 177 & 74.6 \\
\hline \multirow[t]{4}{*}{ Cranial } & 2 & 4 non-coplanar & 123 & 113 & 43.5 \\
\hline & & VMAT arcs & 117 & 113 & 43.5 \\
\hline & & & 58 & 96 & 21.9 \\
\hline & & & 58 & 96 & 21.9 \\
\hline \multirow[t]{2}{*}{ Liver } & 5 & 2 VMAT arcs & 732 & 177 & 74.6 \\
\hline & & & 552 & 177 & 57.1 \\
\hline
\end{tabular}

\section{Results}

\subsection{Validation}

4 For the artificial test case described in section 2.2. and head and neck case, figure 2 shows the 5 difference between the dose rates calculated by the dose rate tool multiplied by their corresponding 6 arc sector times and the static dose distribution calculated by the SMCP. No deviations exceeding the 7 statistical uncertainty of the calculation (about 1\%) were found in any of the test cases.

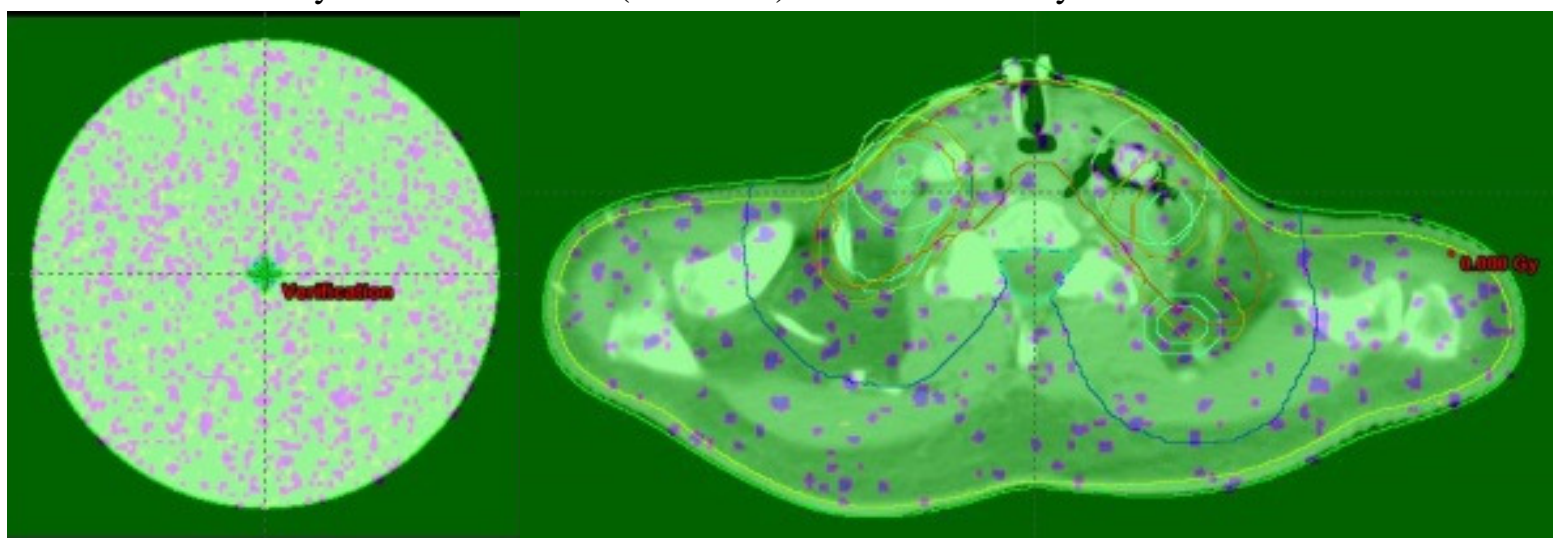

Figure 2: Water phantom and head and neck clinical case with the sum of the dose rate calculation subtracted from static dose, respectively. Color scale from $-0.001 \mathrm{~Gy}$ (magenta) to $+0.001 \mathrm{~Gy}$ (light green)

All plans were applicable without violating any linac specific limitations. Table 3 shows the recorded cumulative MU and treatment time values. Linac reported standard deviations for MU at each CP stayed below 0.1 MU in all applications, while the time at each control point deviated less than $0.4 \mathrm{~s}$ from the calculated values. This indicates machine compliance to the planned MU rates. No treatment prolongation or autonomous alteration of the MU rate by the machine were detected. 
1 Table 3: Treatment delivery records of the three synthetic test cases. For each test case, a fraction was 2 delivered on a Varian Novalis Tx Linac and the Monitor Chamber and time readings recorded from 3 the treatment console. Planned values are as precalculated by the TPS.

\begin{tabular}{|c|c|c|c|c|c|}
\hline \multicolumn{2}{|c|}{ Test case } & \multicolumn{2}{|l|}{ cumula tive $\mathrm{MU}$} & \multicolumn{2}{|l|}{ time $(\mathrm{s})$} \\
\hline 1 & ControtPoint & planned & recorded & planned & recorded \\
\hline & 2 & 600 & 599 & 60.0 & 60.0 \\
\hline & 3 & 1100 & 1103 & 110.0 & 110.4 \\
\hline & 4 & 1500 & 1499 & 150.0 & 150.0 \\
\hline & 5 & 1800 & 1798 & 180.0 & 180.0 \\
\hline & 6 & 2000 & 2000 & 200.0 & 199.8 \\
\hline & & cumula tive $\mathrm{MU}$ & & time $(\mathrm{s})$ & \\
\hline \multirow[t]{7}{*}{2} & ControIPoint & planned & recorded & planned & recorded \\
\hline & 2 & 300 & 296 & 30.0 & 30.0 \\
\hline & 3 & 400 & 401 & 45.0 & 45.0 \\
\hline & 4 & 700 & 701 & 75.0 & 75.0 \\
\hline & 5 & 1700 & 1697 & 175.0 & 174.6 \\
\hline & 6 & 2000 & 2000 & 205.0 & 204.6 \\
\hline & & cumula tive MU & & time $(\mathrm{s})$ & \\
\hline \multirow[t]{7}{*}{3} & ControtPoint & planned & recorded & planned & recorded \\
\hline & 2 & 125 & 123 & 12.5 & 12.6 \\
\hline & 3 & 250 & 249 & 25.0 & 25.2 \\
\hline & 4 & 625 & 621 & 62.5 & 62.4 \\
\hline & 5 & 750 & 745 & 75.0 & 75.0 \\
\hline & 6 & 875 & 871 & 87.5 & 87.6 \\
\hline & 7 & 1000 & 1000 & 100.0 & 100.2 \\
\hline
\end{tabular}

5

6 3.2. Visualization

7 Visualization of this 3D plus time data set poses a challenge, as current TPS are not equipped with 8 time depended dose display routines. Therefore, the calculation tool developed provides a variety of 9 visualization modes, two of which have proven most useful and will be presented here.

10 To get a view of the dose rates expected in a treatment, the tool provides a statistical analysis 11 of dose rate over time, neglecting spatial information in the process. Data is presented as a 12 2D-histogram, showing the number of voxels receiving a certain dose rate in each arc segment. This 13 mode provides fast analysis of the dose rates expected in a treatment or may be applied to a 14 subvolume defined in the treatment structure set.

On the other hand, in 2D plus time mode, the "color wash" dose display mode is mimicked, 16 enhanced by a scrollable time dimension. Instead of dose, dose rates are shown to provide all 17 information about the spatiotemporal variations during the fraction delivery. Example displays for 18 different arc sectors in one slice of the clinical head and neck case are shown in figure 3 . As this mode 19 provides full spatial information, it is better suited for detailed analysis of the dose rate patterns 20 emerging in the treatment. 


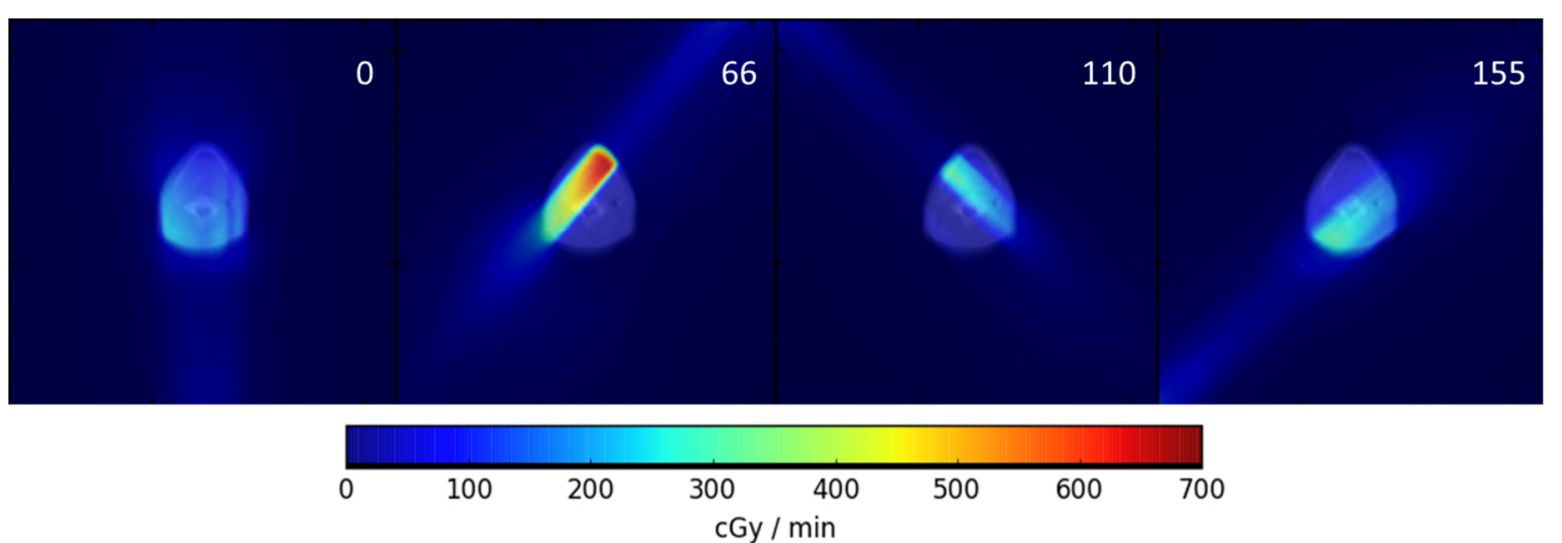

1 Figure 3: Example dose rate distributions of different arc sectors of the first arc of the clinical head 2 and neck VMAT treatment. Dose rates shown in units of $\mathrm{cGy} / \mathrm{min}$ in false color, arc sector numbers 3 shown as white overlay.

\section{3.3. Application}

5 For the three test cases selected, table 4 shows the low dose rates encountered in all VMAT 6 treatments: While maximum dose rates exceed a ratio of $1 \mathrm{cGy} / \mathrm{min}=1 \mathrm{MU} / \mathrm{min}$ to their 7 corresponding sector's MU rate, in each case most of the target volume receives dose rates in the 8 lower end of the dose rate spectra. In the conventionally fractionated head and neck and cranial 9 irradiations, mean PTV dose rate hardly exceeds $100 \mathrm{cGy} / \mathrm{min}$. While being an indicator of the 10 generally low dose rates encountered, mean dose rates are of solely statistical nature as they include 11 both in- and out of field dose rates. Figure 4 provides a histogram of standard deviations of the dose 12 rates calculated for individual voxels over the course of one arc. Especially the higher end of the 13 histogram is of interest here, as this subset of voxels show high variations in dose rates, e.g. if a voxel 14 received high dose rates alternated by very low dose rates. Maximum standard deviations per voxel 15 almost double from the cranial case $( \pm 88.1 \mathrm{cGy} / \mathrm{min})$ to the head and neck case $( \pm 151.0 \mathrm{cGy} / \mathrm{min})$ and 16 again to the hypofractionated liver case $( \pm 299.3 \mathrm{cGy} / \mathrm{min})$.

17 Table 4: Minimal, mean and maximum dose rates of voxels within the Planning Target Volume for 18 three clinical radiotherapy treatment cases.

\begin{tabular}{lllll}
\hline Test case & Arc & $\begin{array}{l}\text { Min. dose rate in } \\
\text { PTV (cGy/min) }\end{array}$ & $\begin{array}{l}\text { Mean dose rate in } \\
\text { PTV (cGy/min) }\end{array}$ & $\begin{array}{l}\text { Max. Dose rate in } \\
\text { PTV (cGy/min) }\end{array}$ \\
\hline Head/Neck & 1 & 0.3 & 70.6 & 424.8 \\
& 2 & 0.2 & 75.6 & 691.4 \\
& 3 & 0.0 & 39.2 & 593.6 \\
& 4 & 0.4 & 47.4 & 318.1 \\
\hline Cranial & 1 & 2.8 & 102.3 & 246.4 \\
& 2 & 2.9 & 76.6 & 215.3 \\
& 3 & 2.9 & 101.6 & 282.7 \\
\hline Liver & 4 & 1.0 & 79.9 & 379.7 \\
& 1 & 5.7 & 249.3 & 772.6 \\
\hline
\end{tabular}




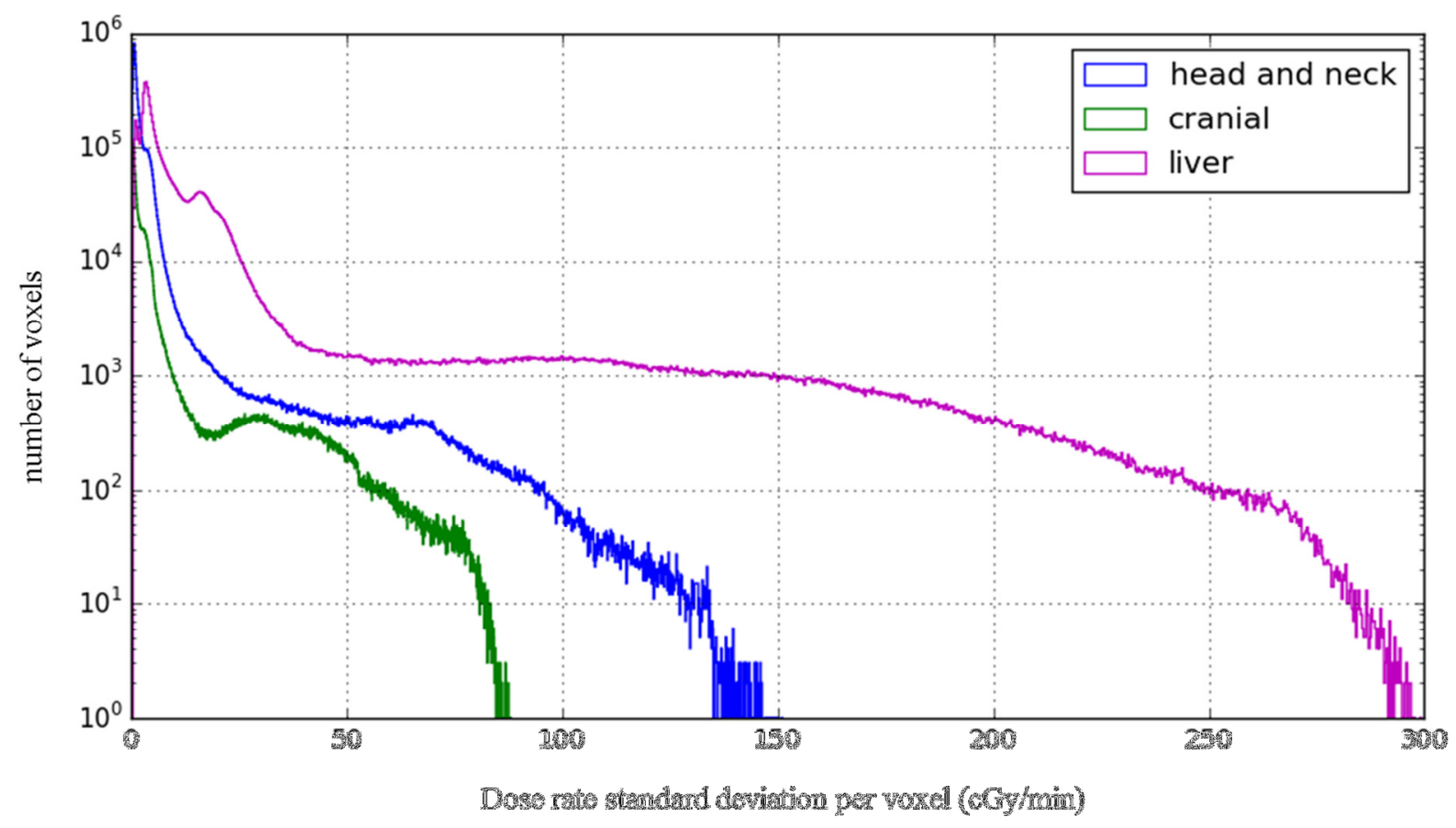

2 Figure 4: Standard deviations of dose rates per voxel of the full calculation volume for the first arc 3 (see table 2 for reference) of the head and neck (blue), cranial (green) and liver (magenta) cases.

4 Standard deviations are calculated individually for each voxel from all dose rates seen by this voxel 5 over the arc considered.

6 Figures 5, 6 and 7 show dose rates (color) and MU rate (black line) in the PTV and an exemplary 7 organ at risk (OAR) of the head and neck, cranial and liver cases selected. For the head and neck and 8 liver irradiation, the spinal cord planning risk volume (PRV, spinal cord expanded by a $2 \mathrm{~mm}$ margin)

9 was chosen as OAR, while the cranial case displays dose rates and MU rates in the brainstem. 
(a) PTV

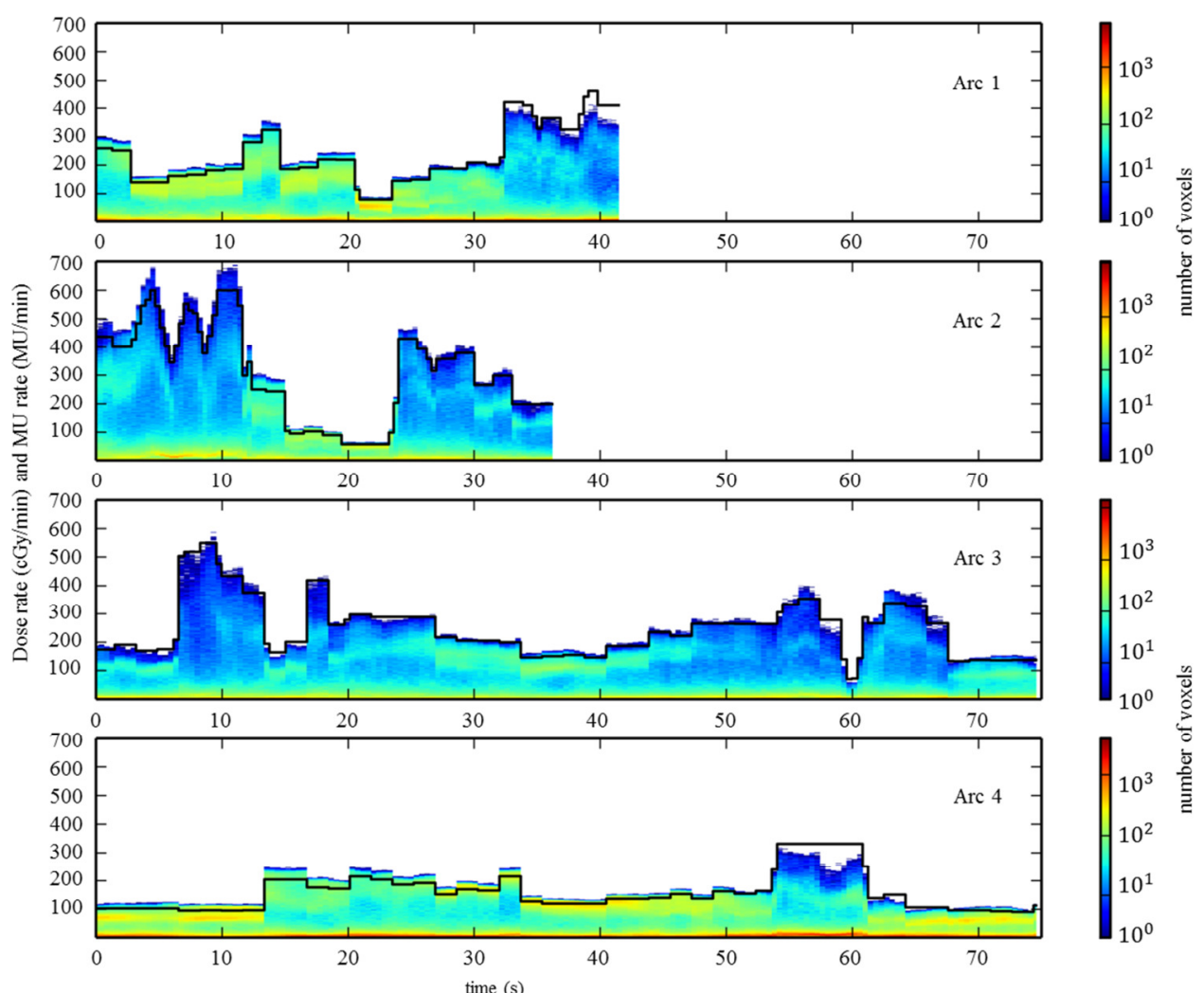

(b) Spinal cord PRV

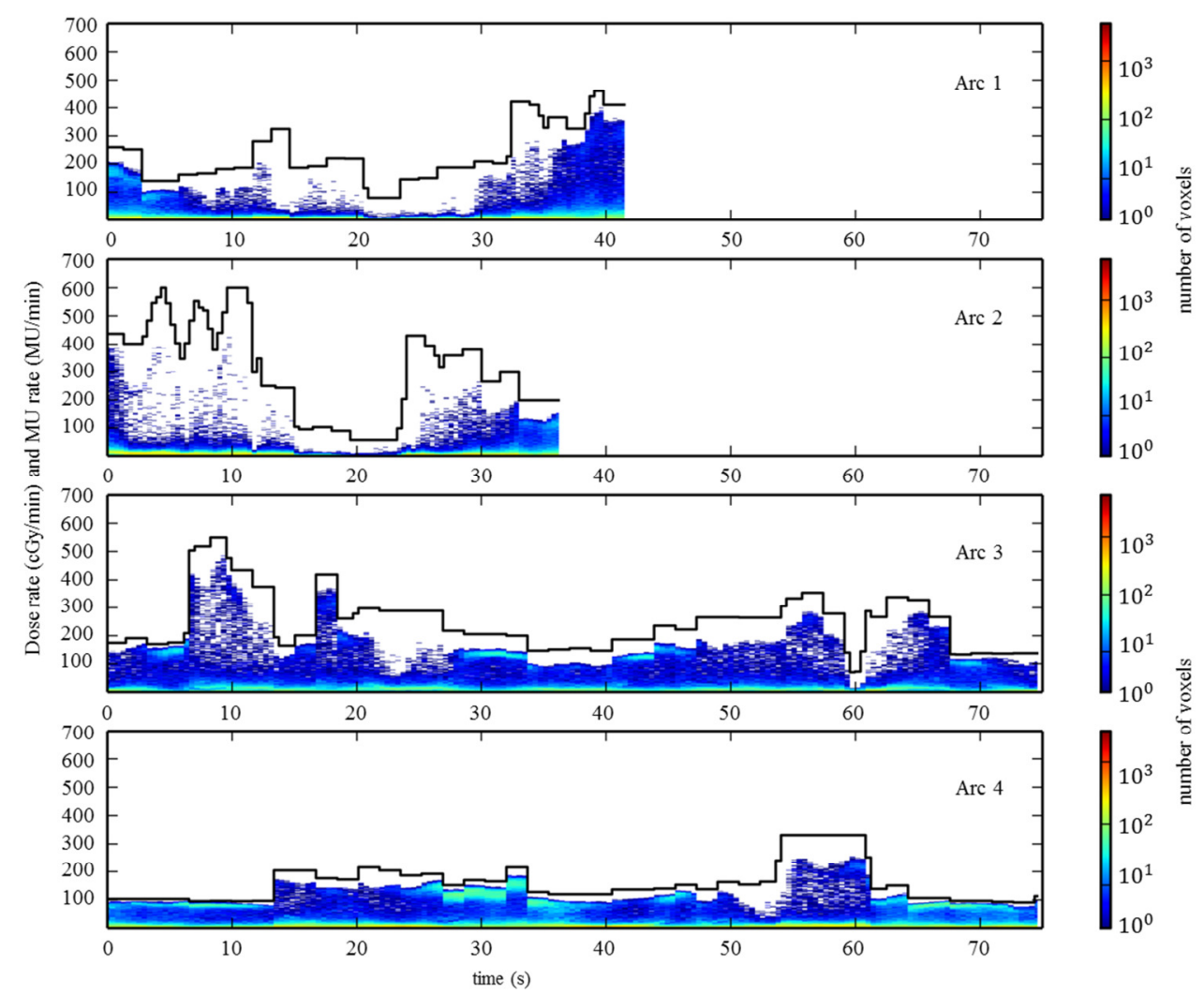

3 Figure 5: 2D histograms of dose rates (color) and MU rate (black line) in voxels of the PTV (a) and 4 spinal cord PRV ( (b), spinal cord expanded by a $2 \mathrm{~mm}$ margin) in a clinical head and neck case over 5 all 4 arcs planned. Number of voxels encoded as color on a logarithmic scale. 
(a) PTV

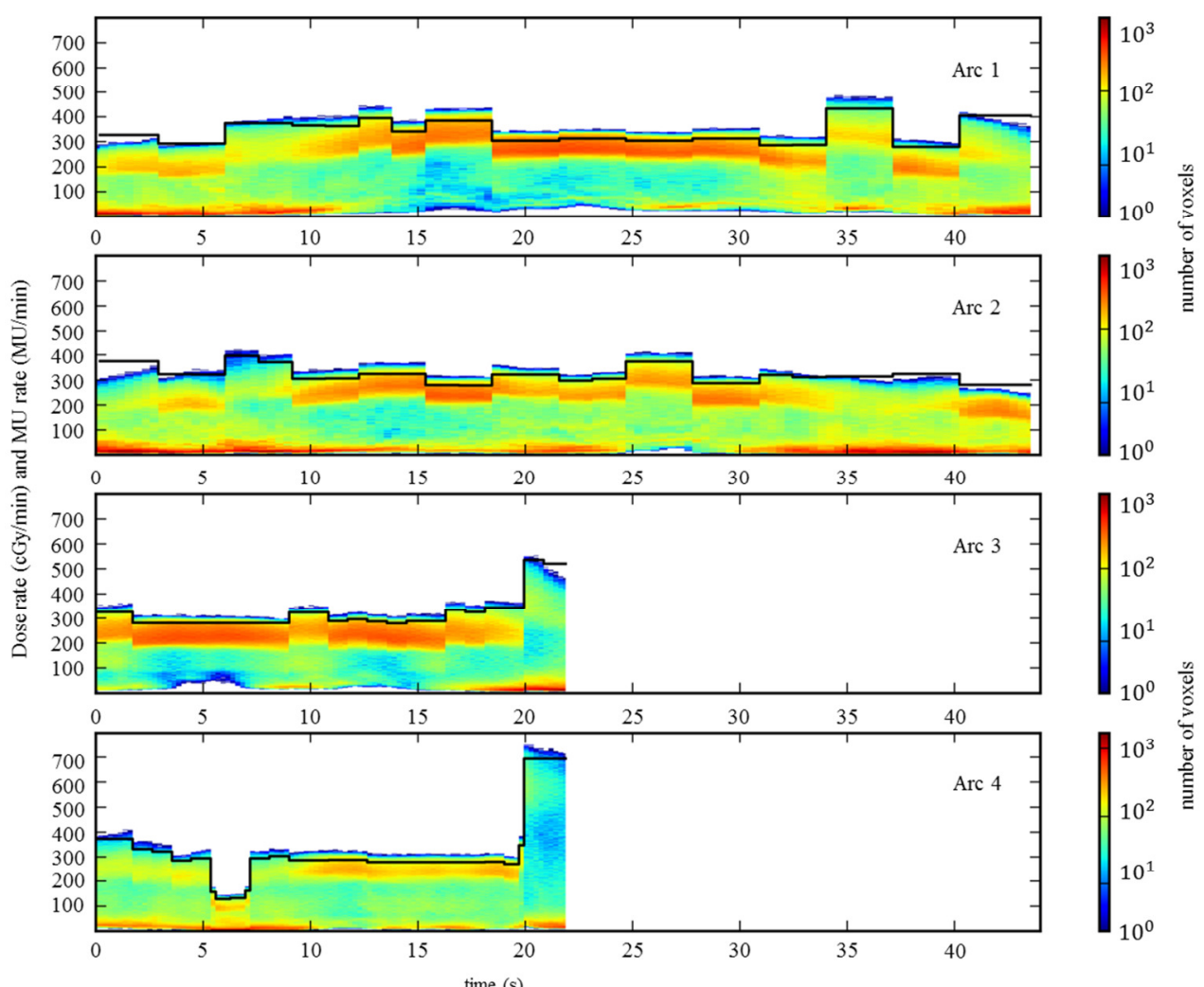

(b) Brain stem

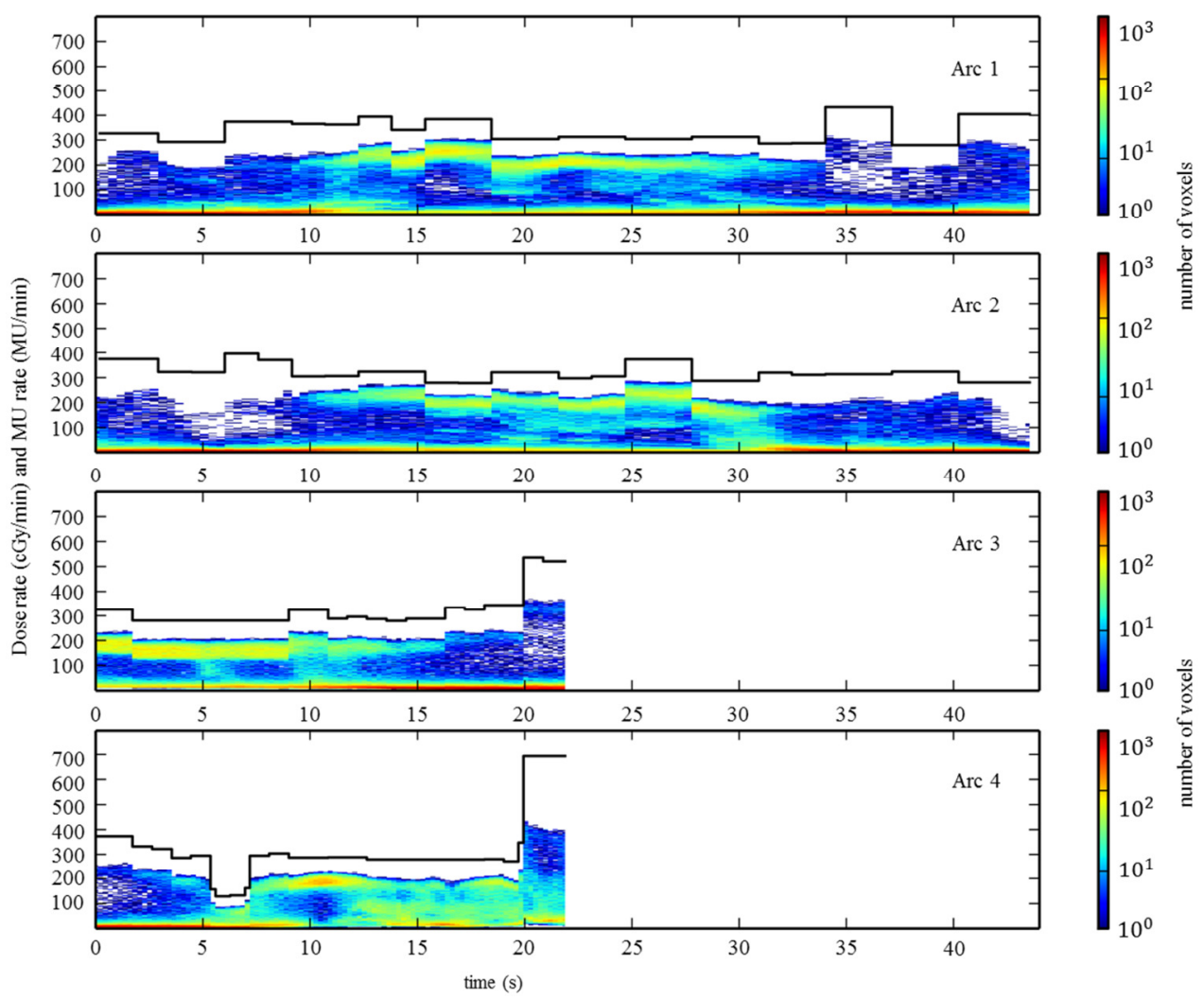

3 Figure 6: 2D histogram of dose rates (color) and MU rate (black line) in voxels of the PTV (a) and 4 brain stem (b) in a cranial irradiation case over all 4 arcs planned. Number of voxels encoded as color 5 on a logarithmic scale. 
(a) PTV

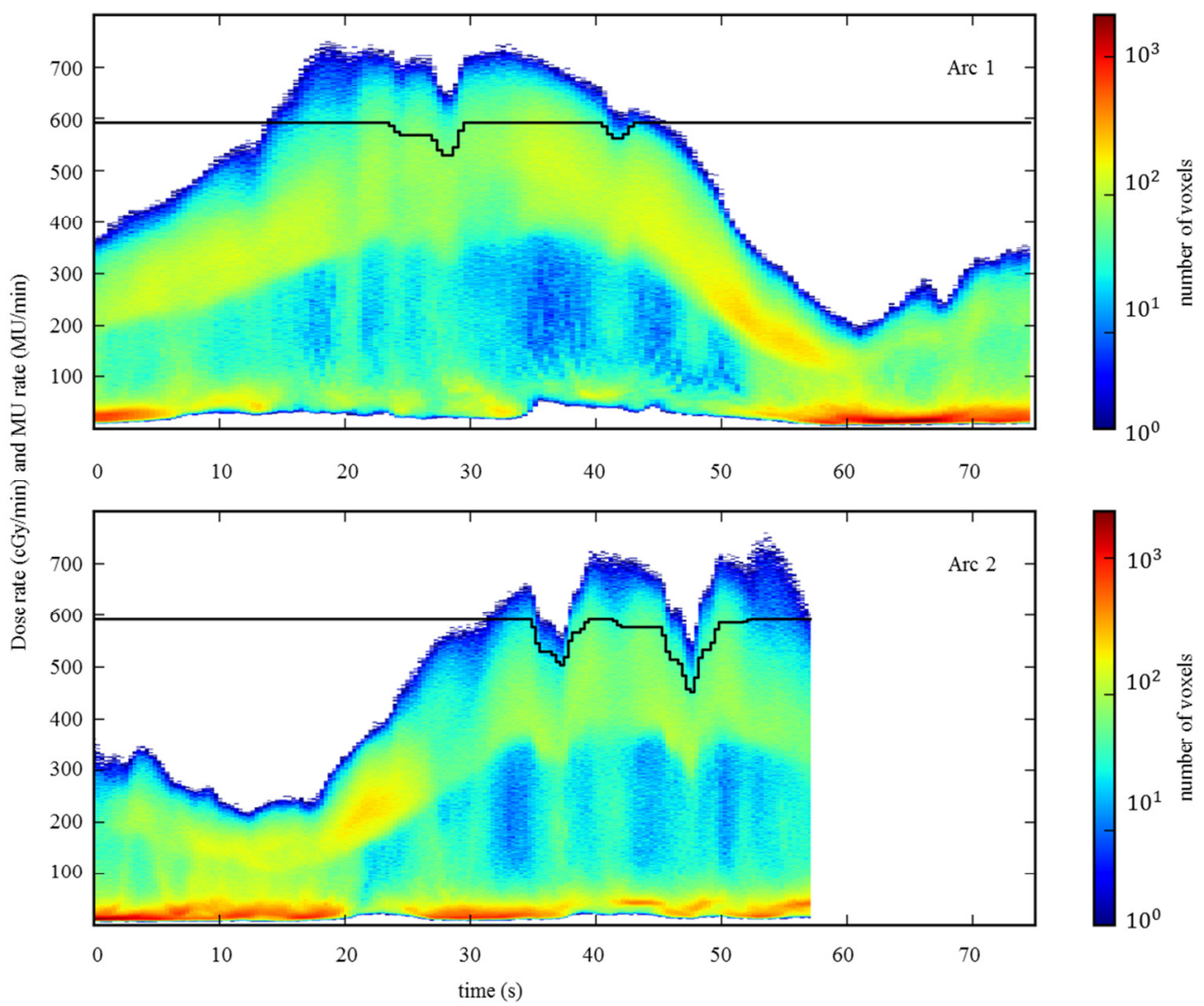

(b) Spinal cord PRV

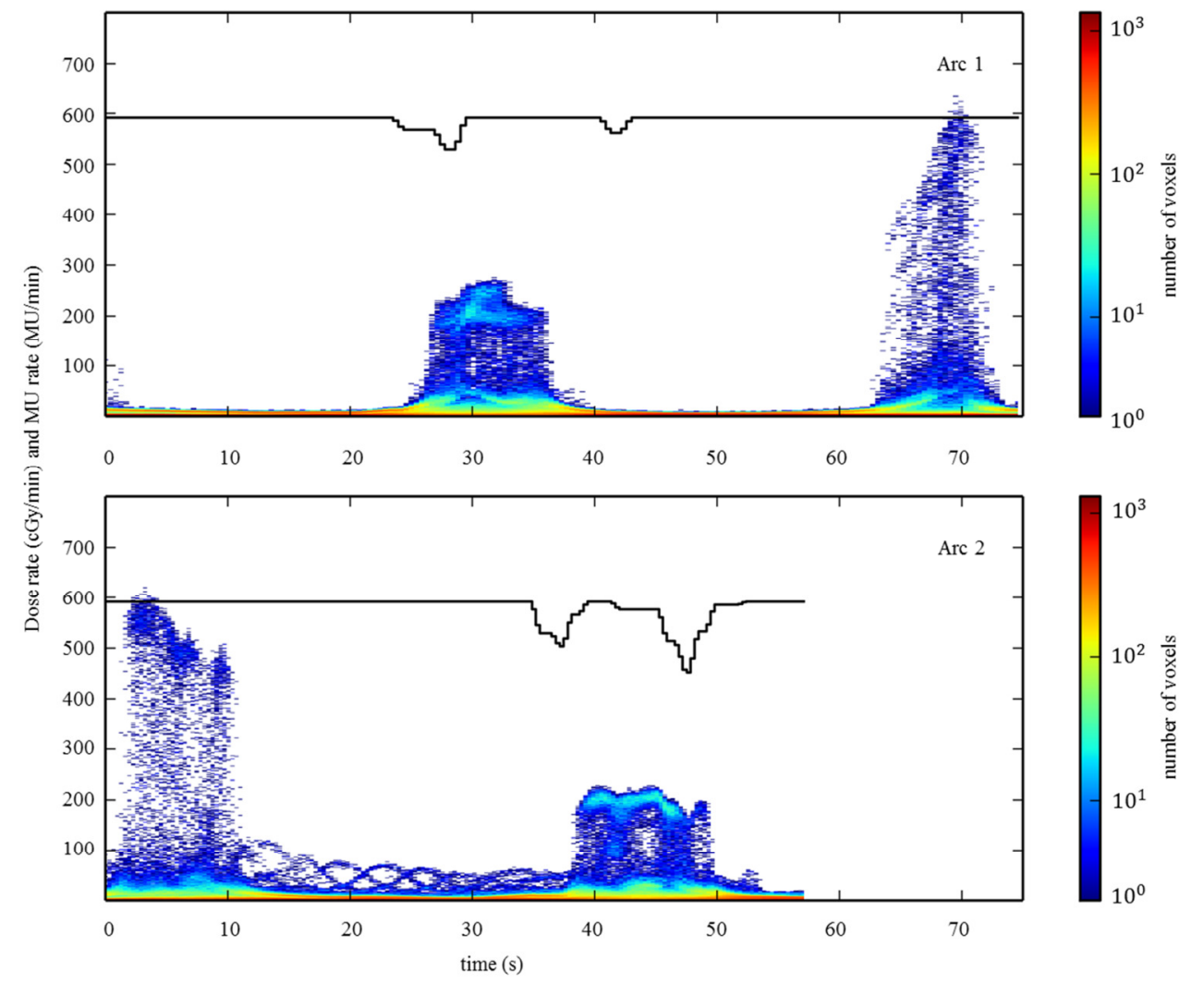

3 Figure 7: 2D histogram of dose rates (color) and MU rate (black line) in voxels of the PTV (a) and 4 spinal cord PRV (b) in a hypofractionated liver case over all 2 arcs planned. Number of voxels 5 encoded as color on a logarithmic scale. 
1 In order to catch small, but important, dose rate values per voxel contributing most of the dose of this 2 voxel per arc, figures 8 and 9 show weighted dose rate histograms of the PTV and spinal cord PRV of 3 the hypofractionated liver case. Each dose rate value was multiplied by the fraction of dose the 4 corresponding arc sector contributed to the total (per arc) dose of the corresponding voxel. Both 5 figures show a contribution of a broad spectrum of dose rates to the dose distribution.

PTV

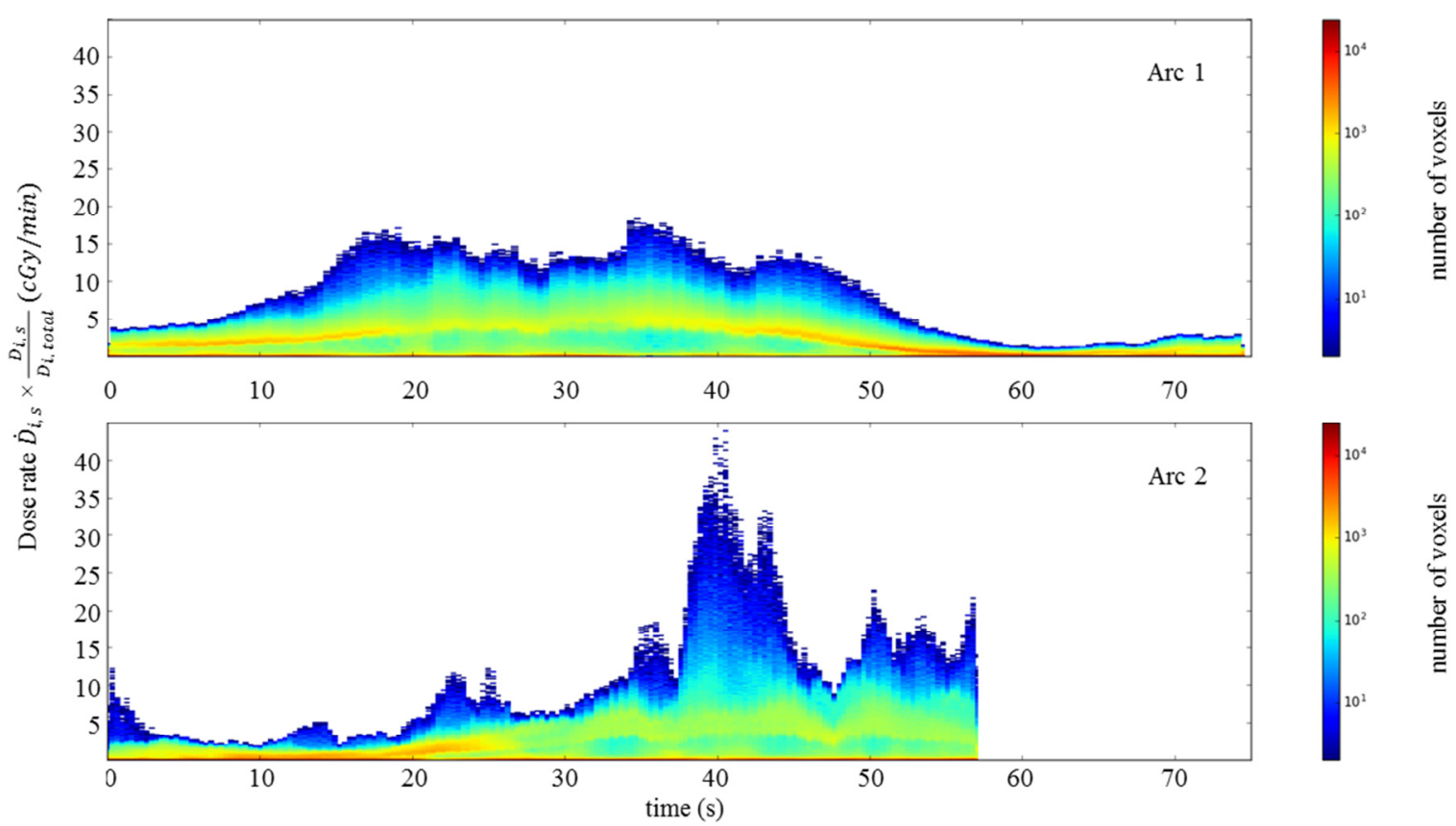

7 Figure 8: Weighted dose rate histogram in the PTV of the hypofractionated liver case. Each dose rate $8 \dot{D}_{i, s}$ in voxel $i$ and sector $s$ was multiplied by the fraction of dose $\frac{D_{i, s}}{D_{i, \text { total }}}$ that sector $s$ contributes to 9 the total (per arc) dose of voxel $i$. 


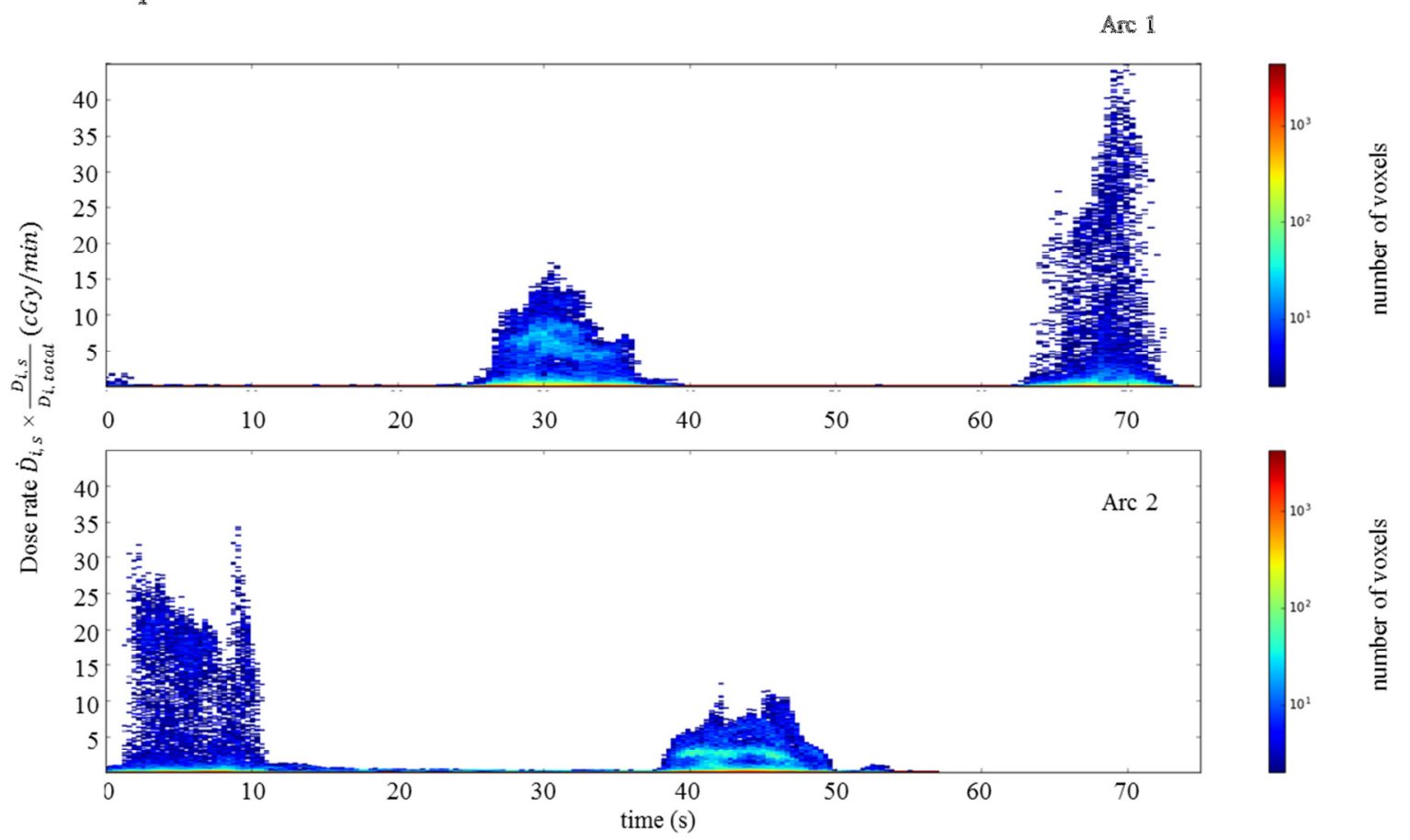

2 Figure 9: Weighted dose rate histogram in the spinal cord PRV of the hypofractionated liver case.

3 Each dose rate $\dot{D}_{i, s}$ in voxel $i$ and sector $s$ was multiplied by the fraction of dose $\frac{D_{i, s}}{D_{i, \text { total }}}$ that sector $s$

4 contributes to the total (per arc) dose of voxel $i$.

5 Both the head and neck as well as the cranial test case feature MU rate modulation in blocks of

6 several arc sectors with dose rates following the pattern. On the contrary, the hypofractionated liver

7 case modulates both the MU rate and dose rate without any visible grouping. OAR dose rates

8 generally tend to be lower than those encountered in the PTV, while still showing maximum dose

9 rates at in-field heights in all of the test cases. In the liver case, the body diameter permits sparing of

10 the spinal cord for most of the two arcs. Most of the dose to the OAR is delivered by two high dose

11 rate peaks, showing the case of the spinal cord lying within the beam on the entry and exit side,

12 respectively. For the head and neck and cranial cases, maximum dose rates to the OAR rarely drop

13 below $100 \mathrm{cGy} / \mathrm{min}$, despite exhibiting lower MU- and maximum dose rates per voxel. OAR dose

14 rates are within a smaller range than for the liver case, illustrated by the banding of medium dose rate

15 voxels in the OAR histograms of the head and neck and cranial cases.

\section{4. Discussion}

17 A new efficient tool was developed to analyze dose rates per arc sector using MC calculations.

18 Following the depth dose curve, dose rates vary from values expected at reference conditions, with a

19 small fraction of voxels even surpassing a one to one ratio between dose rate in cGy/min and MU rate

20 in MU/min at skin depths smaller than $10 \mathrm{~cm}$. Despite a maximum MU rate set to $600 \mathrm{MU} / \mathrm{min}$, analysis of the clinical test cases using conventional fractionation showed a substantial fraction of voxels receiving dose rates below $100 \mathrm{cGy} / \mathrm{min}$. The setting of MU rate in a VMAT radiotherapy plan merely defines a point of reference in a spectrum of dose rates encountered. Not only may the machine lower the MU rate according to its limitations in VMAT treatments, rendering dose rates encountered dependent on the number of arcs and gantry angle travelled (as between the 2-arc and 4arc sample cases). But also looking at the clinical test cases revealed that only a small part of the voxels in the target volume is irradiated with dose rates nearing a ratio of $1 \mathrm{cGy} / \mathrm{min}=1 \mathrm{MU} / \mathrm{min}$ 
1 even below the set maximum MU rate. The more complex the target shape and modulation of the field shape, the larger the fraction of voxels irradiated at low dose rates becomes as shown by the clinical head and neck case selected. These findings may have substantial implications for future research. On one hand, when assessing relative effectiveness of different dose rates in VMAT, radiobiological data correlated to the dose rates encountered needs to be applied. If research should show a reduced effectiveness of lower dose rates or even a threshold for altered effectiveness in low dose rates as it was described by Hall (Hall 1972) for cobalt irradiation, weighting of low dose rates might be adjusted accordingly. Some dose rates may even be low enough for DNA repair to outweigh radiation damage. This would change radiobiological effectiveness during and after phases of low dose rate irradiation, possibly even affecting effectiveness of high dose rates applied afterwards. On the other hand, application of the tool may resolve conflicting evidence. Radiobiological models may be verified and clinical findings explained by the new insights provided.

All of the clinical treatment plans considered show arc sector times in the order of $0.2-0.4 \mathrm{~s}$, achieving sub-second resolution of the calculated dose rate distributions, but still suffering from averaging. The dose rates shown leverage the DICOM convention to keep dose rate constant between two consecutive control points even in modulated treatments. Recordings of synthetic test cases confirmed that MU rates are not being interpolated, but kept constant between control points. Similar to partial volume effects, voxels changing their dose rate between control points due to movement are assigned an averaged dose rate, leading to "partial time effects" in areas of high dose rate gradients. Further, dose rates reported are calculated values only, focusing on planning stage. Similar to the calculation of dose distributions, any deviations between planned and delivered dose rates, caused for example by the linac MU feedback loop, are not considered in this work. The tool developed focuses on VMAT as dose rate variations are more emphasized in this technique compared to IMRT, but its algorithm is applicable to both step and shoot and sliding-window IMRT as well. There definitely is a need to further improve resolution of dose rate analysis. So far, dose rate can be plotted with respect to time, but not tissue. Dose rate effect is believed to vary with tissue (Favaudon et al 2014, Steel et al 1986) and should thus be weighted accordingly between e.g. tumor tissue and organs at risk. So far, the tool can only give dose rate distributions for each organ separately, but not visualize them together. These may be used to provide a "deconvolution" of the spatio-temporal patterns of dose rates in VMAT to apply fixed dose rate evidence to these de-facto modern standard treatment techniques. It would be interesting to see radiobiological and clinical research interpret these values. In clinical routine, these data could aid deciding between treatment modalities of different dose rates, such as flattened versus flattening filter free beams or conventional versus hypofractionation. With the advent of new fractionation schemes, knowledge about dose rates would allow to choose a fractionation scheme tailored to the dose rates encountered in specific regions of interest. Radiation effect to the tumor could be tailored according to dose rate models as could damage to each organ at risk individually. As modelling of dose rate response evolves (Herr et al 2014), this tool serves as an insight into dose rates encountered in modulated radiotherapy treatments and hopes to aid and guide further investigation.

\section{5. Conclusion}

41 A tool to analyze planned dose rate distributions with sub-second accuracy was successfully developed and validated. Visualization is done via histogram distributions of dose rates in regions of interest over a treatment fraction or 2D plus time display as color wash. Dose rates per arc segment encountered in clinical VMAT cases show a continuous spectrum of dose rates with a mean value in the lower part of the spectrum for both conventional fractionation and for a hypofractionated liver case. Dose rate distributions in modulated radiotherapy can now be assessed, and will serve as input for further radiobiological study. In the future, these data could aid deciding between treatment 
modalities of different dose rates, such as conventional versus hypofractionation. Fractionation schemes might even be tailored to the dose rates encountered in specific regions of interest.

Almond P R and Biggs P J 1999 AAPM' s TG-51 protocol for clinical reference dosimetry of highenergy photon and electron beams 1847-70

Altman M B, Chmura S J, Deasy J O and Roeske J C 2006 Optimization of the temporal pattern of radiation: an IMRT based study. Int. J. Radiat. Oncol. Biol. Phys. 66 898-905 Online: http://www.ncbi.nlm.nih.gov/pubmed/17011463

Bewes J M, Suchowerska N, Jackson M, Zhang M and McKenzie D R 2008 The radiobiological effect of intra-fraction dose-rate modulation in intensity modulated radiation therapy (IMRT). Phys. Med. Biol. 53 3567-78 Online: http://www.ncbi.nlm.nih.gov/pubmed/18560049

Favaudon V, Caplier L, Monceau V, Pouzoulet F, Sayarath M, Fouillade C, Poupon M-F, Brito I, Hupé P, Bourhis J, Hall J, Fontaine J-J and Vozenin M-C 2014 Ultrahigh dose-rate FLASH irradiation increases the differential response between normal and tumor tissue in mice. Sci. Transl. Med. 6 245ra93 Online: http://www.ncbi.nlm.nih.gov/pubmed/25031268

Fix M, Manser P and Frei D 2007 An efficient framework for photon Monte Carlo treatment planning Phys. Med. ... 425 N425-37 Online: http://iopscience.iop.org/0031-9155/52/19/N01

Fowler J F, Welsh J S and Howard S P 2004 Loss of biological effect in prolonged fraction delivery. Int. J. Radiat. Oncol. Biol. Phys. 59 242-9 Online: http://www.ncbi.nlm.nih.gov/pubmed/15093921

Hall E J 1972 Radiation dose-rate: a factor of importance in radiobiology and radiotherapy $B r . J$. Radiol. 45 81-97

Herr L, Friedrich T, Durante M and Scholz M 2014 A model of photon cell killing based on the spatio-temporal clustering of DNA damage in higher order chromatin structures. PLoS One 9 e83923 Online: http://journals.plos.org/plosone/article?id=10.1371/journal.pone.0083923

Joiner M C, Mogili N, Marples B and Burmeister J 2010 Significant dose can be lost by extended delivery times in IMRT with x rays but not high-LET radiations. Med. Phys. 37 2457-65 Online: http://www.ncbi.nlm.nih.gov/pubmed/20632556

Ling C C, Gerweck L E, Zaider M and Yorke E 2010 Dose-rate effects in external beam radiotherapy redux. Radiother. Oncol. 95 261-8 Online: http://www.ncbi.nlm.nih.gov/pubmed/20363041

Mu X, Löfroth P-O, Karlsson M and Zackrisson B 2003 The effect of fraction time in intensity modulated radiotherapy: theoretical and experimental evaluation of an optimisation problem. Radiother. Oncol. 68 181-7 Online: http://www.ncbi.nlm.nih.gov/pubmed/12972314

Murphy M J, Lin P-S and Ozhasoglu C 2007 Intra-fraction dose delivery timing during stereotactic radiotherapy can influence the radiobiological effect Med. Phys. 34481 Online: http://scitation.aip.org/content/aapm/journal/medphys/34/2/10.1118/1.2409750

Ogino H, Shibamoto Y, Sugie C, Ito M and Fractionation R 2005 Biological Effects of Intermittent Radiation in Cultured Tumor Cells : 46 401-6

Ponette V, Le Péchoux C, Deniaud-Alexandre E, Fernet M, Giocanti N, Tourbez H and Favaudon V 2000 Hyperfast, early cell response to ionizing radiation. Int. J. Radiat. Biol. 76 1233-43 Online: http://www.ncbi.nlm.nih.gov/pubmed/10993634

Shibamoto Y, Ito M, Sugie C, Ogino H and Hara M 2004 Recovery from sublethal damage during intermittent exposures in cultured tumor cells: implications for dose modification in radiosurgery and IMRT. Int. J. Radiat. Oncol. Biol. Phys. 59 1484-90 Online: http://www.ncbi.nlm.nih.gov/pubmed/15275736

Steel G G, Down J D, Peacock J H and Stephens T C 1986 Dose-rate effects and the repair of radiation damage. Radiother. Oncol. 5 321-31 Online: 
http://www.ncbi.nlm.nih.gov/pubmed/3726169

2 Wang J Z, Li X A, D'Souza W D and Stewart R D 2003 Impact of prolonged fraction delivery times on tumor control: a note of caution for intensity-modulated radiation therapy (IMRT). Int. J. Radiat. Oncol. Biol. Phys. 57 543-52 Online: http://www.ncbi.nlm.nih.gov/pubmed/12957268 